\title{
Effects of intracoronary arterial injection of tirofiban on no-reflow phenomenon in patients with acute ST-segment elevation myocardial infarction underwent primary percutaneous coronary intervention
}

\author{
Hongyu Duan, Xiping Wan, Bing Li \\ Department of Cardiology, The Third Affiliated Hospital of Inner Mongolia Medical University, Baotou, Inner Mongolia, China
}

Received: February 23, 2017

DOI: $10.14725 /$ dcc.v4n2p1

Accepted: March 28, $2017 \quad$ Online Published: June 10, 2017

URL: http://dx.doi.org/10.14725/dcc.v4n2p1

\begin{abstract}
Objective: To compare the effect of intracoronary arterial injection of tirofiban and sodium nitroprusside (SNP) on no-reflow phenomenon in patients with acute ST-segment elevation myocardial infarction (STEMI) underwent primary percutaneous coronary intervention (PCI).

Methods: A total of 68 patients with acute STEMI who had no-reflow phenomenon during PCI were chosen and randomly divided into SNP group $(\mathrm{n}=34)$ and tirofiban group $(\mathrm{n}=34)$. Aiming at no-reflow phenomenon during PCI, with the use of microcatheters, intracoronary arterial injection of tirofiban was given in tirofiban group, while intracoronary bolus of SNP was given in SNP group. Coronary angiography was conducted to record TIMI flow grade of the infarct-related artery after 10 minutes. Plasma brain natriuretic peptide (BNP) was monitored before and after PCI (in 24 hours). With the help of ultrasound cardiogram, left ventricular ejection fraction (LVEF), left ventricular end-diastolic dimension (LVEDD) and left ventricular endsystolic dimension (LVESD) were recorded and compared in 30 days after PCI.

Results: The ratio of TIMI grade 3 blood flow in tirofiban group was significantly higher than that in SNP group [76.5\% (26/34) vs. 52.9\% (18/34), $p=.03$ ]. BNP levels in two groups had no statistical significance before PCI ( $p=.16)$, but in $24 \mathrm{~h}$ after PCI, BNP levels in tirofiban group were significantly lower than those in SNP group $[(439.00 \pm 4.90) \mu \mathrm{mol} / \mathrm{L}$ vs. $(632.00 \pm 3.63)$ $\mu \mathrm{mol} / \mathrm{L}, p=.02]$. In 30 days after PCI, LVEF, LVEDD and LVESD in tirofiban group were all superior to those in SNP group (all $p<.05$ ).

Conclusions: Intracoronary arterial injection of tirofiban was superior to SNP in improving no-reflow phenomenon in STEMI patients after PCI in the emergency treatment. Tirofiban therapy can increase coronary blood flow and myocardial perfusion after the occurrence of no-reflow phenomenon during PCI in STEMI patients, and improve long-term prognosis.
\end{abstract}

Key Words: Angioplasty, Transluminal, Percutaneous coronary, No-reflow phenomenon, Tirofiban, Sodium nitroprusside

Percutaneous coronary intervention (PCI) is an effective means in the treatment of acute ST-segment elevation myocardial infarction (STEMI). ${ }^{[1]}$ PCI can quickly and effec- tively open the infarct-related artery (IRA), restore the effective myocardial reperfusion, improve left ventricular dysfunction and reduce mortality. When STEMI occurs, IRA is

\footnotetext{
*Correspondence: Hongyu Duan; E-mail: duanhongy-2@163.com; Address: Department of Cardiology, The Third Affiliated Hospital of Inner Mongolia Medical University, Baotou, Inner Mongolia, China.
} 
often prone to be thrombotic. PCI can make blood clots to shed off and cause thromboembolism at the terminal microcirculation, and it probably results in no-reflow phenomenon, i.e., myocardial tissues in the area supported by IRA have no perfusion or bad perfusion, which leads to microcirculation disturbance, affects the efficacy of PCI, and becomes an independent risk factor of long-term sudden cardiac death. ${ }^{[2]}$ Tirofiban, a receptor antagonist of platelet glycoprotein IIb/IIIa, has a strong inhibitory effect on platelets. It can reduce thrombus formation burden in the coronary artery during surgery and secondary distal microcirculation thromboembolism, effectively improve myocardial perfusion, increase IRA recanalization rate and improve patients' prognosis. Research results from Wang et al. ${ }^{[3]}$ showed that intracoronary arterial injection of tirofiban can significantly improve blood flow in the infarct-related artery and myocardial perfusion, without increasing bleeding complications. Sodium nitroprusside (SNP) has effects on improving endothelial function and microcirculation spasm. Intracoronary bolus of SNP can effectively improve no-reflow phenomenon in patients with STEMI during PCI in the emergency treatment. ${ }^{[4]}$ In this study, intracoronary arterial injections of tirofiban and SNP were conducted respectively with the use of microcatheters to STEMI patients who needed PCI in the emergency treatment. It was intended to compare the effects of two drugs on no-reflow phenomenon during PCI.

\section{Objects and methods}

\subsection{Research objects}

68 cases of STEMI patients, who had no-reflow phenomenon during PCI in the emergency treatment during May 2013 to May 2016, were chosen from Department of Cardiology in the Third Affiliated Hospital of Inner Mongolia Medical University as subjects. The chosen patients were randomly divided into tirofiban group $(\mathrm{n}=34)$ and SNP group $(n=34)$. Tirofiban group was consisted of 19 males and 15 females, aged 35 to 75 years, with an average age of $(66.2 \pm 7.3)$ years; SNP group contained 18 males and 16 females, aged 42 to 75 years, with an average age of $(63.3 \pm 8.5)$ years. Inclusion criteria were as follows: (1) The diagnosis conformed to STEMI diagnostic criteria established by American College of Cardiology and American Heart Association (ACC/AHA). (2) The coronary angiography of posterior IRA showed that flow loss occurred on the distal portion of the lesion (TIMI grade 0-1, noreflow), without intimal tear, dissection, thromboembolism and other mechanical obstruction. (3) The blood pressure was controlled and kept at desired levels. Exclusion criteria were as follows: (1) Patients with serious infection, as well as severe cardiopulmonary, hepatic and renal insufficiency. (2) Patients with platelet or coagulation system dysfunction, hemorrhagic \& hematological diseases, as well as anticoag- ulation contraindications. (3) Patients who had an allergy to tirofiban and SNP. All subjects were required to sign informed consent forms, and the scheme was approved by Ethics Committee of Scientific Research Task in the Third Affiliated Hospital of Inner Mongolia Medical University.

\subsection{Methods}

\subsubsection{Therapeutic scheme}

After admission, all patients in the therapeutic scheme immediately received physical examinations such as 18 lead ECG, routine blood test, bleeding \& coagulation (time) test, blood analysis, myocardial enzyme test etc., and the blood pressure was required to be controlled under 160/100 $\mathrm{mmHg}$; all patients were required to be given adequate rest, sedation therapy, oxygen inhalation treatment, pain management, ECG monitoring and preoperative preparation. All patients were required to take and chew enteric-coated aspirin $300 \mathrm{mg}$ and clopidogrel $300 \mathrm{mg}$ before PCI. After PCI, low molecular weight heparin (LMWH) $5000 \mathrm{U}$ was used as a routine application by subcutaneous injection of $\mathrm{q} / 12 \mathrm{~h} / 7 \mathrm{~d}$. Aiming at no-reflow phenomenon during PCI, intracoronary arterial injection of tirofiban was given in tirofiban group (10 $\mu \mathrm{g} / \mathrm{kg}$, in 1-3 min, and continue to pump for $36-48 \mathrm{~h}$ at the rate of $\left.0.15 \mu \mathrm{g} \cdot \mathrm{kg}^{-1} \cdot \mathrm{min}^{-1}\right)$ and SNP was given in SNP group (100 $\mu \mathrm{g}$, bolus injection in $2 \mathrm{~s})$.

\subsubsection{Determination of indicators}

With the use of microcatheters, intracoronary aryerial injection of tirofiban was given in tirofiban group, while intracoronary bolus of SNP was given in SNP group. Coronary angiography was conducted to record TIMI flow grade of the infarct-related artery after 10 minutes. BNP was monitored before and after PCI (in 24 hours). With the help of ultrasound cardiogram, left ventricular ejection fraction (LVEF), left ventricular end-diastolic dimension (LVEDD) and left ventricular end-systolic dimension (LVESD) were recorded in 30 days after PCI.

\subsection{Statistical methods}

The measurement data were represented by $\bar{x} \pm \mathrm{s}$, while the categorical data were represented by percentage composition ratio. SPSS 19.0 statistical package was applied to statistical treatment. The measurement data were compared by use of $t$-test, while the categorical data were compared by means of $\chi^{2}$ test. The difference $(p<.05)$ was of statistical significance. 


\section{Results}

\subsection{Comparison in the number of cases of thrombus aspiration and balloon dilatation in two groups}

Patients in two groups had no thromboembolism or dissection during PCI. Thrombus aspiration and balloon dilatation can increase the possibility that causes blood clots to shed off and thromboembolism at the terminal microcirculation, which will lead to slow flow or no-reflow in the coronary artery. In this study, tirofiban group can be compared with
SNP group in the ratio of thrombus aspiration (52.9\% vs. $\left.44.1 \%, \chi^{2}=0.38, p=.16\right)$ and the ratio of balloon dilatation $\left(47.1 \%\right.$ vs. $\left.38.2 \%, \chi^{2}=0.24, p=.17\right)$.

\subsection{Comparison of baseline data in two groups}

Differences in baseline morbidity (hypertension, diabetes, hyperlipoidemia etc.) and premedication of two groups had no statistical significance (all $p>.05$, see Table 1 ).

Table 1: Comparison of baseline morbidity (hypertension, diabetes, hyperlipoidemia etc.) and premedication in two groups $[\mathrm{n}(\%)]$

\begin{tabular}{lllll}
\hline & Tirofiban Group $(\mathbf{n}=\mathbf{3 4})$ & SNP Group $(\mathbf{n}=\mathbf{3 4})$ & $\chi^{\mathbf{2}}$ & $p$-value \\
\hline Hypertension & $17(50.0)$ & $11(32.4)$ & 2.19 & .07 \\
Diabetes & $7(20.6)$ & $10(29.4)$ & 0.71 & .16 \\
Hyperlipoidemia & $10(29.4)$ & $8(23.5)$ & 0.30 & .19 \\
Medication & & & & .21 \\
- $\beta$-receptor blocker & $30(88.2)$ & $28(82.4)$ & 0.47 & .16 \\
- ACEI (ARB) & $27(79.4)$ & $30(88.2)$ & 0.98 & .21 \\
\multicolumn{1}{c}{ Statins } & $29(85.3)$ & $27(79.4)$ & 0.40 & \\
\hline
\end{tabular}

Table 2: Comparison of infarction positions and CK-MB levels in two groups

\begin{tabular}{lllll}
\hline & Tirofiban Group $(\mathbf{n}=34)$ & SNP Group $(\mathbf{n}=\mathbf{3 4})$ & $\chi^{2} / \mathbf{t}$-value & $\boldsymbol{p}$-value \\
\hline Infarction Position [n $(\%)]$ & & & & \\
- Anteroseptal & $5(14.7)$ & $4(11.8)$ & 0.00 & .77 \\
- Anterior & $8(23.5)$ & $6(17.7)$ & 0.36 & .81 \\
- Extensive Anterior & $7(20.6)$ & $8(23.5)$ & 0.09 & .48 \\
- Inferior & $9(26.5)$ & $10(29.4)$ & 0.21 & .21 \\
- Inferior + Strictly Posterior + Right Ventricular & $5(14.7)$ & $6(17.6)$ & 0.11 & .24 \\
CK-MB $(\bar{x} \pm \mathrm{s}, \mathrm{U} / \mathrm{L})$ & $115.2 \pm 26.9$ & $109.0 \pm 39.5$ & 3.43 & .02 \\
\hline
\end{tabular}

Table 3: Comparison of TIMI flow grade of the infarct-related artery [n (\%)]

\begin{tabular}{lllll}
\hline & $\mathbf{n}$ & TIMI 3 & TIMI 2 & TIMI 0-1 \\
\hline SNP Group & 34 & $18(52.9)$ & $13(38.3)$ & $3(8.8)$ \\
Tirofiban Group & 34 & $26(76.5)$ & $7(20.6)$ & $1(2.9)$ \\
$\chi^{2}$ & & 4.12 & 2.55 & 0.27 \\
$p$-value & & .03 & .06 & .25 \\
\hline
\end{tabular}

\subsection{Comparison of infarction positions and myocar- dial enzyme indicators in two groups}

Differences in infarction positions and myocardial enzyme indicators of two groups had no statistical significance (all $p>.05$, see Table 2).

\subsection{Comparison of TIMI flow grade of the infarct- related artery in two groups}

Coronary angiography results showed that the ratio of TIMI 3 flow in tirofiban group was higher than that in SNP group, and the difference was of statistical significance $(p<.05$, see Table 3).

\subsection{Comparison of plasma BNP levels in two groups}

In 24 hours after PCI, BNP level in tirofiban group was significantly lower than that in SNP group [(439.00 \pm 4.90$)$ $\mu \mathrm{mol} / \mathrm{L}$ vs. $(632.00 \pm 3.63) \mu \mathrm{mol} / \mathrm{L}]$, and the difference was of statistical significance $(t=1.94, p=.02)$. 
Table 4: Comparison of LVEDD, LVESD and LVEF in two groups in 30 days after PCI $(\bar{x} \pm \mathrm{s})$

\begin{tabular}{lllll}
\hline & $\mathbf{n}$ & LVEDD $(\mathbf{m m})$ & LVESD $(\mathbf{m m})$ & LVEF $(\%)$ \\
\hline SNP Group & 34 & $54.64 \pm 5.11$ & $48.04 \pm 2.74$ & $53.71 \pm 5.42$ \\
Tirofiban Group & 34 & $50.58 \pm 2.62$ & $42.58 \pm 5.19$ & $59.96 \pm 3.19$ \\
$t$-value & & 3.16 & 2.79 & 3.62 \\
$p$-value & & .03 & .04 & .02 \\
\hline
\end{tabular}

\subsection{Comparison of LVESD, LVEDD and LVEF in two groups}

In 30 days after PCI, LVEF, LVEDD and LVESD in tirofiban group were all superior to those in SNP group, and differences were of statistical significance $(p<.05$, see Table 4$)$.

\section{Discussion}

The therapeutic principle of STEMI is to open IRA in the early stage. It is intended to reperfuse ischemic myocardia, narrow the area of myocardial necrosis and improve prognosis. At present, PCI in the emergency treatment is the primary therapy for opening culprit vessels and curing STEMI. However, the occurrence of no-reflow phenomenon during PCI significantly affects patients' prognosis. Even if appropriate medication is given during surgery, the clinical prognosis in patients is worse than that in patients without no-reflow phenomenon. Therefore, to improve myocardial microcirculation reperfusion and reduce the incidence of noreflow phenomenon has become a new hot spot in the study of coronary intervention.

Currently, the pathogenesis of no-reflow in the coronary artery has not been completely identified yet. However, it has been generally acknowledged that microcirculation damage or dysfunction will arise consequently. At present, possible pathogenesis are as follows: (1) thromboembolism on the distal portion of the coronary artery; (2) interstitial and intracellular edema; (3) coronaryspasm; (4) capillary collapse; (5) platelet aggregation; (6) production of oxygen free radicals; (7) coagulation cascade reactions; (8) mechanical compression and hyperglycemia; (9) hyperlipoidemia and other factors. ${ }^{[5]}$

In the event that no-reflow phenomenon occurs during PCI and develops rapidly, the severe will go into shock. It is

\section{References}

[1] Cui XH, Wang JX, Fan CM, et al. Clinical analysis of emergency percutaneous coronary intervention in 156 patients with acute ST segment elevation myocardial infarction. Chin J Intervent Cardiol. 2010(6); 345-347.

[2] Bolad IA, Khan B, Ghumman W. Contrast-induced coronary no flow required to cope with it as soon as possible. The principle is to maintain hemodynamic stability and dilate coronary capillaries, in the effect that reperfusion of myocardial capillaries can be restored rapidly. ${ }^{[6]}$ A large amount of clinical practices and researches showed that anti-platelet aggregation for the purpose of preventing thrombosis is an effective method to improve prognosis. ${ }^{[7]}$ This research is intended to explore the clinical effect of tirofiban on STEMI patients with no-reflow phenomenon during PCI. The ratio of TIMI grade 3 flow (infarct-related artery) in tirofiban group was significantly higher than that in SNP group. Therefore, tirofiban can improve TIMI flow grade after no-reflow phenomenon occurs, and restore effective perfusion in the coronary artery as soon as possible. Before PCI, the difference in BNP levels of two groups had no statistical significance, which indicated that differences in the degree of myocardial damage and cardiac function had no statistical significance; in 24 hours after PCI, BNP level in tirofiban group was obviously lower than that in SNP group, which showed that tirofiban had a protective effect on myocardial damage caused by no-reflow phenomenon. Currently, BNP is a generally accepted predictive index of myocardial damage and cardiac failure. In 30 days after PCI, LVEF, LVEDD and LVESD in tirofiban group were all superior to those in SNP group, which indicated that tirofiban can relieve thrombus formation burden, improve IRA reperfusion, and then improve left ventricular function and prognosis consequently. Therefore, tirofiban is a preferable strategy to cope with noreflow phenomenon during PCI in the emergency treatment and worthy of being applied clinically. The research is limited as the number of specimens is not large and the observation time is not long. It remains to be further verified in the large-scale clinical research.

\section{Conflicts of Interest Disclosure}

The authors have no conflicts of interest related to this article.

phenomenon during diagnostic coronary angiography. Texas Heart Inst J. 2011; 3895: 600-607.

[3] Wang HL, Xing SY, Dong PS, et al. Safety and efficacy of intracoronary tirofiban administration in patients with serious thrombus burden and ST-elevation myocardial infarction undergoing percntaneous coronary intervention. Eur Rev Med Phannaeol Sci. 2014; 18(23): 3690-3695. 
[4] Miao LF, Yang D, Huang CL, et al. Effects of Intracoronary administration of nitroprusside on no-reflow phenomenon during emergency percutaneous coronary intervention in patients with acute myocardial infarction. J Clinc Cardiol. 2008; 24(10): 765-769.

[5] Galasso G, Schiekofer S, Danna C, et al. No-reflow phenomenon: pathophysiology, diagnosis, prevention, and treatment. A review of the current literature and future perspectives. An geology. 2014; 65(3): 180-189. https://doi.org/10.1177/ 0003319712474336
[6] Deng XZ, Yan P. Progress in the study of coronary slow flow phenomenon in traditional Chinese and Western Medicine. Shenzhen Journal of Integrated Traditional Chinese and Western Medicine. 2014; 24(1): 120-122.

[7] He XP, Su SH, Ye JF, et al. Influence of intravascular tirofiban injection in occlusion site of coronary artery on patients with acute myocardial infarction complicated with diabetes mellitus. Chin J Cardiovasc Med. 2016; 21(3): 231-235. 